\title{
Prevalence of Hepatitis B Virus Infection Among the Out-patientsin Oyo,Oyo State Nigeria
}

\section{Jo Olayiwola and Lanlehin Folake*}

Department of Biological Sciences, Ajayi Crowther University Oyo, Oyo State, Nigeria

*Corresponding Author: Lanlehin Folake, Department of Biological Sciences, Ajayi Crowther University Oyo, Oyo State, Nigeria.
Received: September 25, 2021

Published: October 23, 2021

(C) All rights are reserved by Jo Olayiwola and Lanlehin Folake.

\begin{abstract}
Hepatitis B virus (HBV) infection is considered to be the ninth leading cause of death, is one of the major public health problems in the world. Nigeria was rated $8.3 \%$ prevalence of hepatitis. Hepatitis virus also called jaundice is an inflammation of the liver caused by DNA virus belonging to the genus of Orthohepadnavirus from the family of hepadnaviridea and sub family of Retroviridae. In consequence of this, there is need to investigate the prevalence of hepatitis B viral infection in Oyo state. Informed consent of the out-patients attending clinics in Oyo State were obtained and questionnaires were administered to investigate their knowledge and attitude towards safer practices against the spread of HBV. The study design was a cross-sectional descriptive study of consented individuals of both (Male and Female), the study place were Oyo and Ibadan, Nigeria. The study was conducted for the period of three months. The seroprevalence of the participants were examined with LabAcon rapid kit with 98.2\% - 100\% and 97.2\% - 99.8 for specificity and sensitivity respectively. Out of 45 female participants, five (5) were seropositive to hepatitis B surface antigen (HBsAg) in whole blood and the relationship is significant (4.15), this study shows a low prevalence of hepatitis B viral infection in Oyo State, Nigeria.
\end{abstract}

Keywords: Hepatitis B Virus; Infection; Diagnosis; Prevalence

\section{Introduction}

\section{Hepatitis B virus infection}

Chronic hepatitis B virus (HBV) infection is considered to be the ninth leading cause of death, is one of the major public health problems in the world. As it is one of the main causes of liver disease which is responsible for about 360 million cases of chronic hepatitis [1]. Hepatitis B virus is an highly infectious agent and is about 75-200 times more infectious than HIV, this is because HBV does not depend only on body fluid like HIV as a means of transmission, but it can also be transmitted by aerosols [2]. Hepatitis which is called Jaundice [3] is an inflammation of the liver caused by a DNA virus belonging to the genus of Orthohepadnavirus, fam- ily of Hepadnaviride and subfamily of Retroviridae. It can be transmitted through sharing of sharp objects, blood transfusion of infected patient, vertical transmission, sexual transmission, it can be transmitted through direct contact with infected patient body fluid like sweat, saliva, blood etc.

Basically the spread of hepatitis B among children is through vertical transmission that is from mother to the baby [4]. Household items such as toothbrush, razor, and needle are also potential fomites which serve as means of transmission. Exposure to contaminated or unsterilized surgical equipment from medical and dental procedures has been implicated in the transmission of the virus. Community practice involving body and ear piercing, tattoo- 
ing of the body, and the use of unsterilized object for traditional rituals, circumcision and face marking are major risk factor in the community transmitted hepatitis infection [5]. This infection result in chronic cases like liver cirrhosis, liver cancer and liver failure [4].

Some of the clinical signs and symptoms include fatigue, sudden nausea and vomiting, abdominal pain especially on the upper right side under the lower ribs, loss of appetite, dark urine and joint pain. The prevalence of HBV in Nigeria has brought about proper management and the implementation of effective preventive measures. The main preventive measure that has been carried out in Nigeria to prevent this infection is through routine vaccination of newborns because children are born without a fully developed natural immune system [6]. This study is to investigate the prevalence of hepatitis B among people attending community health care in Oyo Town and also investigate the knowledge of the subjects about hepatitis $B$, attitude and practice of safe procedures against the transmission of HBV. The statement of the problem according to [7], Hepatitis is considered the ninth leading cause of death and also one of the major public health problems in the world. Nigeria with a prevalence rate of $8.1 \%$, the prevalence of hepatitis B virus in Oyo, Oyo State Nigeria, is still not known. Therefore, the spread of theses virus posed a threat to health and more research is needed to know the prevalence rate. The study significance is to know the prevalence rate of hepatitis B virus in Oyo, Oyo State Nigeria, this will help health practitioners and the population at large to carry out preventive methods to curb the spread of hepatitis B virus and also help health practitioners to carry out necessary sensitization and awareness about hepatitis B virus.

Justification of this study is due to the few data regarding the prevalence of hepatitis B virus among out-patients in Oyo, Oyo State Nigeria. Through the findings of this study, we intends to update the limited knowledge about the prevalence of hepatitis B virus which is crucial for health workers to know the prevalence of hepatitis B virus and also provide control measures in the spread of the virus. The objective aim of the study is to investigate the prevalence of hepatitis B virus among Out-Patients in Oyo, Oyo State Nigeria.

\section{Methods and Materials \\ Study area}

Oyo town is a city in Oyo state, Nigeria, founded in 1830s. And known to its people as the New Oyo (Oyo Atiba) to distinguish it from the former capital to the north which had been deserted as a result of rumours of war. On the $28^{\text {th }}$ May, 1987 the ministry of health states that Oyo town as fifty-two health outlet within the state. The study area of this study would be under Oyo state public and state hospitals

\section{Ethical clearance}

Ethical approval number obtained for the conduction of the research is FNS/EA/NO/2020/001.

\section{Study period}

The study comprises of sample collection, treatment and analysis was conducted for the period of three months.

The criterion for inclusion as subjects was the acceptance through consent.

\section{Study design}

The study design was a cross-sectional descriptive study. The sample population comprises of male and female age group (18 50 ). which was obtained by offering consent form to the intending participants. This consent was sought after the concept of research was explained and due signed by the participant who is regarded as consent. A structured closed-ended questionnaire was administrated to these subjects and was guided by explaining the unclear terms to them before filling the questionnaires. The response of the respondent were imputed into excel page for the analysis. The excel package used for this study is Microsoft Excel 2010.

\section{Population \\ Study population}

At least a total number of 50 blood samples of consented individuals both (Male and Female) were Oyo and Ibadan, Nigeria.

\section{Study subject}

Patients on attendance at the public state and private hospitals for cases different from hepatitis cases were primarily targeted. However, consented community individuals were included for the Hepatitis B surveillance.

\section{Administration of questionnaire}

Individuals participating in the survey were subjected to an interviewer administered questionnaire. The questionnaire focusing on personal vaccination of individual, the mother was asked about the child's personal and vaccination history including the mother, 
father, the household's characteristics and socioeconomic status. The questionnaire did not include any sensitive or embarrassing questions. Interviews were conducted in study participant's respective health care centre, hospital, offices, house and church. The questionnaire was administered by the study investigator participating in the survey. The study investigator would systematically go over the questions and distribute the questionnaire to the study participant and if the prospective study participant happens to be an illiterate, the questionnaire is then read out to them and the study investigators participating in the survey help to fill the questionnaire based on the prospective study participant response. This was expected to help in proper collection of questionnaire and to minimize inter observer and responder bias, especially because both respondent and investigator did not know their serological status at the time the interview was conducted.

\section{Sample collection}

This process involve the taking of blood sample from an individual making use of a needle, tourniquet, anticoagulant bottle, hand gloves, cotton wool and alcohol.

\section{Transportation of sample}

This is the process of taking the blood sample from the clinic down to the laboratory for proper analysis. During this process, the sample transporter wears his/her laboratory coat, hand glove and facemask, the blood sample is then checked and recorded accordingly based on the clinic code and laboratory code. The sample is then transported in a cold chain temperature so as to prevent the sample from lysing before getting to the laboratory.

\section{Sample analysis}

For the sample analysis, LabAcon rapid kit was used. to test the samples for hepatitis B antibodies, it is a rapid chromatographic immunoassay for the qualitative detection of antibodies to hepatitis B virus in serum or plasma. The specificity and sensitivity of LabAcon rapid kit is $98.2 \%-100 \%$ and $97.2 \%$ - 99.8 respectively (Blumberg, 1971).

\section{Results}

Statistical analysis of the data will be done using Statistical package for social sciences (SPSS) version 2.0. The Statistical tools used in the study includes percentage distribution and chi-square test.

Samples were collected from Ajayi Crowther University health centre Oyo and Ojoo health centre Ibadan. A total of fifty (50) sam- ples were collected comprising of forty-five (45) samples from female and five (5) from male subjects. All the participants are within the age range of 18years - 50 years.

The occurrence of hepatitis B virus was observed to be 5 out of 45 female investigated in this study. However, only 5 male subjects were involved in the investigation without any positive results (Table 1). The relationship between the gender and knowledge of HBV in the study population revealed that there was association between these two variables. The level of significance is 0.546 which is greater than standard value of 0.05 (Table 2).

\begin{tabular}{|l|c|c|c|}
\hline Gender & + & - & Total \\
\hline M & 0 & 5 & 5 \\
\hline F & 5 & 40 & 45 \\
\hline Total & 5 & 45 & 50 \\
\hline
\end{tabular}

Table 1: Prevalence of Hepatitis B virus based on the gender.

The chi square value obtained is 4.15 compared to chi square table value of 3.84 therefore with $95 \%$ certainty, we can reject the null hypothesis and conclude that according to our data prevalence of HBV is related to gender.

\begin{tabular}{|l|c|c|c|c|c|}
\hline Gender & Yes & No & Don't know & & $\begin{array}{c}\text { Level of } \\
\text { Significance }\end{array}$ \\
\hline Male & & & 7 & 2 & 10 \\
\hline Female & 12 & 9 & 3 & 34 & \\
\hline Total & 19 & 11 & 4 & 44 & 0.546 \\
\hline
\end{tabular}

Table 2: Investigation of the sex of the participants and their knowledge of HBV.

In this case, the p-value (0.546) is greater than the standard alpha value (0.05), the relationship between the two variables (sex and knowledge of HBV) is insignificant.

\section{Discussion}

A total number of 50 blood samples were collected between the age range $18-50$ yars, $45(90 \%)$ were females and $5(10 \%)$ were male. 5 of the participant who happened to be female were positive for HBsAg giving a seroprevalence of $4.0 \%$, from the test carried out, it can be said that the female gender are the most prevalence patient that visit the clinic on a regular basis compared 
to the male gender. From the statistical analysis of the questionnaire administered, the relationship in knowledge between sex that is the male and female gender shows that the result is insignificant, meaning that the knowledge of hepatitis B virus regarding sex is not based on the type of gender.

The knowledge exhibited towards HBV between male and female participants showed the evidence that sex does not reveal any difference in the exposure to awareness to the information as regards prevalence, control, transmission and pathogenesis of the virus.

\section{Recommendation}

The government and health organization should raise the awareness of hepatitis B viral infection for the population and the populace should be encourage to check their hepatitis B status and those who happens to be negative to HBsAg should be encourage to get a vaccine against the viral infection while those that are positive to HBsAg should be encourage to visit their doctor for treatment and management of the viral infection.

\section{Conclusion}

Although HBV infection remains a serious health problem in $\mathrm{Ni}$ geria due to the prevalence of the viral infection. This study shows a low prevalence of hepatitis B viral infection in Oyo State, Nigeria. But the awareness and attitude of the population in regard to this viral infection in Oyo State, Nigeria have a high positive rate which if not attended to will have a positive effect on the prevalence of hepatitis B virus in Oyo State, Nigeria.

\section{Bibliography}

1. World health organization. "Hepatitis b and c, immunization, vaccines and biologicals" (2013).

2. Samaranayake LP. "Essential microbiology for dentistry" (1998).

3. https;//www.webmd.com

4. WHO. "Guidelines for the prevention, care and treatment of persons with chronic hepatitis b infection". Geneva, Switzerland: WHO (2015).

5. "Global surveillance and control of hepatitis c. report of a who consultation organized in collaboration with the viral hepati- tis prevention board, Antwerp, Belgium". Journal of Viral Hepatitis 6 (1999): 35-47.

6. Sadoh AE and Sadoh WE. "Serological markers of hepatitis b infection in infants presenting for their first immunization". Nigerian Journal of Paediatrics 40 (2013): 248-253.

7. WHO. Department of communicable diseases surveillance and response. hepatitis $\mathrm{B}$.

Volume 4 Issue 11 November 2021 (C)All rights are reserved by Jo Olayiwola and Lanlehin Folake.

Citation: JO Olayiwola and Lanlehin Folake. "Prevalence of Hepatitis B Virus Infection Among the Out-patients in Oyo, Oyo State Nigeria". Acta Scientific Microbiology 4.11 (2021): 62-65. 